Archives de sciences sociales des religions

169 | Janvier-mars 2015

Philosophie et religion

\title{
Introduction - Philosophie et religion
}

\section{Vincent Delecroix}

\section{OpenEdition}

Journals

Édition électronique

URL : https://journals.openedition.org/assr/26602

DOI : $10.4000 /$ assr.26602

ISSN : 1777-5825

Éditeur

Éditions de l'EHESS

Édition imprimée

Date de publication : 5 juin 2015

Pagination : 13-19

ISBN : 978-2-7132-2468-3

ISSN : 0335-5985

Référence électronique

Vincent Delecroix, «Introduction - Philosophie et religion », Archives de sciences sociales des religions [En ligne], 169 | Janvier-mars 2015, mis en ligne le 01 juin 2018, consulté le 21 septembre 2021. URL http://journals.openedition.org/assr/26602 ; DOI : https://doi.org/10.4000/assr.26602 
Philosophie et religion 



\section{Vincent Delecroix}

\section{Introduction \\ Philosophie et religion}

Où en est la philosophie de la religion aujourd'hui ? C'est pour tenter de répondre à cette question qu'on a tâché d'offrir au lecteur un panorama, forcément partiel, de son paysage actuel - essentiellement sa partie française - avec le souci d'en montrer la diversité d'objets, de méthodes, de problématiques, mais aussi les liens qui s'y établissent éventuellement - et le conflit ou le malentendu est aussi une sorte de lien - avec les sciences sociales du religieux.

À tort ou à raison, ces dernières ont pu considérer avec une certaine méfiance l'exercice de la philosophie de la religion. Trop de "métaphysique », trop d'abstraction et de généralités, d'essentialisme, trop de christiano-centrisme, trop de compromission avec l'objet également et de cryptothéologie ; ou, à l'inverse, trop de critique naïve ou d'idéologie positiviste, trop d'insensibilité aussi : une formule commode et ressassée répète que les philosophes parlent du religieux comme les sourds de la musique. Il n'est pas d'ailleurs jusqu'au terme de Philosophie de la religion (au singulier) qui n'illustre selon elles le défaut congénital qu'elle a hérité malheureusement des circonstances de sa naissance : on ne saurait mieux témoigner de son lien funeste avec la nature métaphysique de la Religionsphilosophie qui a servi de creuset à l'idéalisme allemand et qui du reste prétendait ouvertement réaliser la synthèse de la « raison » et de la « révélation ». Il n'est même pas sûr que substituer commodément le pluriel au singulier dans un tel syntagme témoigne à l'inverse d'une heureuse prise de conscience de la diversité des traditions religieuses. Ni qu'elle témoigne d'un heureux renoncement à cette tâche désuète qu'elle s'était prescrite : produire le concept de la Religion, déterminer l'essence de la Religion.

Comme on le sait peut-être, la philosophie de la religion n'est pas non plus en reste dans la critique des sciences sociales et de l'indigence conceptuelle des approches prétendument "empiriques » - c'est-à-dire qu'elle n'est pas non plus en reste dans la pratique de la caricature ou du préjugé. Il lui est par ailleurs assez facile de faire la preuve de son intérêt jamais démenti pour la "réalité concrète ", comme on dit, des phénomènes religieux et de revendiquer par exemple une véritable sensibilité, d'ailleurs fondatrice, à l'histoire des religions 
ou à l'analyse des pratiques. Il y a du reste de bonnes raisons qui pourraient à l'inverse légitimer le fait de traiter "abstraitement» de la religion. Il est vrai qu'elle a pu sembler partir de très loin en la matière, si l'on se souvient qu'elle fut au départ dominée par des couples analytiques tels que religion naturelle/ religions positives ou religion/superstition ; ou encore grevée par le projet, explicite ou non, non pas tant de se rendre intelligible la religion que de bâtir une religion rationnelle dont l'une des conditions d'ailleurs, dans le prolongement du déisme, était qu'elle fût débarrassée le plus possible des pratiques rituelles. Mais elle a également, et dès le début, toujours su réagir à ses propres réductionnismes, ce qui explique notamment la variété des traditions qui l'innervent ( $\mathrm{du}$ reste cette variété tient également aux rapports qu'elle entretient, ou refuse d'entretenir, avec les autres sciences du religieux). On peut même dire qu'un certain nombre de griefs qui lui ont été faits durant son histoire, elle se les était d'abord formulés à elle-même, par exemple celui de se focaliser dangereusement sur les seules représentations religieuses, au détriment du culte ou des pratiques, ou, corrélativement d'ailleurs, de ne saisir que les formes « rationalisées » des religions, c'est-à-dire finalement ce qui les rend affines à un système philosophique : le souci de revenir à la "religion vivante " comme à son objet véritable, par exemple, contre les conceptions abstraites (ou « rationalisantes »), la prétention à saisir «l'expérience » religieuse dans sa radicale originalité constituent une marque de fabrique, si l'on peut dire, de la phénoménologie de la religion - et d'ailleurs pour le pire comme pour le meilleur.

Mais on n'a ici ni la place ni le loisir d'énoncer l'ensemble de ses griefs mutuels, dont le catalogue permettrait pourtant d'établir combien un « dialogue » entre la philosophie de la religion et les sciences sociales du religieux, pour autant qu'il serait souhaitable, ne va pas de soi. On n'a pas cherché non plus par cette présentation à montrer combien ces griefs ont pu ou pourraient être infondés, pas plus d'ailleurs qu'on n'a voulu démontrer que le conflit (ou l'incompréhension, ou la défiance) était terminé - on verra du reste que pour certains l'ignorance mutuelle ou une incompréhension résolue paraît encore la meilleure solution. On a en revanche voulu montrer à la fois l'originalité de cet exercice et son dynamisme actuel, sa diversité et sa fécondité. Et, peut-être, sa nécessité.

Dans l'introduction de ses Leçons sur la philosophie de la religion, en effet, Hegel revient avec profit sur les besoins auxquels pouvaient répondre un tel exercice. Si l'on entend par là que la philosophie de la religion répond toujours à quelque chose comme une exigence de la raison, laquelle du reste ne se réduit pas, mais c'est déjà beaucoup, au seul souci d'intelligibilité et du moins implique toujours un principe critique au titre de principe fondateur de son exercice, on aurait évidemment tort d'en oublier le caractère historique et même social : la raison qui s'y exerce n'est jamais indépendante de ces situations et de ces configurations historiques, elle les exprime. Quelque chose, donc, dans une époque et 
dans le phénomène religieux lui-même vient solliciter cet exercice, demande à être pensé, apprécié ou évalué par la « raison » philosophique. Mais cette raison à son tour connaît des configurations historiques qui déterminent à la fois sa position à l'égard des phénomènes religieux et ses intérêts en la matière. Les développements, les méthodes, les objets mêmes de la philosophie de la religion répondent donc à la fois à des logiques internes à l'histoire de la philosophie et même à l'histoire de la raison et à des sollicitations extérieures.

L'une des contributions essentielles que Hegel a fournie à cette discipline - quand bien même on en refuserait solidement l'encombrant patronage - est de l'avoir indissociablement liée à un retour réflexif sur elle-même, qui l'oblige à s'interroger sur sa signification et sa nécessité historiques : elle ne peut pas s'exercer sans tâcher de penser simultanément pourquoi il y a de la philosophie de la religion et pourquoi elle prend telle forme particulière, pourquoi elle découpe tel objet dans le champ du phénomène religieux, pourquoi telle dimension de ce phénomène constitue pour elle un problème. C'est ce qui fait que ses développements, dont on verra ici la diversité, fournissent aussi par eux-mêmes de précieuses indications sur l'état de notre modernité, pour autant que celle-ci puisse se comprendre par un certain état des rapports entre la raison et les religions et pour autant que la philosophie de la religion exprime particulièrement cet état. Mais elle n'est pas seulement un symptôme ou un indice de cet état : si elle continue d'être l'un des lieux essentiels où se traite le problème des rapports entre raison et religions - et cette raison doit être entendue aussi bien comme raison politique ou encore comme raison morale que comme raison cognitive si elle exprime dans ce traitement les préoccupations essentielles de la raison face à l'expérience et l'institution religieuses, elle est aussi et par là même un analyseur de la modernité tardive dans laquelle nous vivons.

Quel peut être aujourd'hui son projet, au regard notamment des développements des sciences du religieux? "Penser la religion " est une formulation qui a le mérite trompeur de l'innocuité : au mieux elle a, dans son indétermination, peu de sens; au pire elle trahit la vanité d'un projet à la fois globalisant et substantialisant, l'illégitimité de sa prétention et la nullité de son résultat. Elle se révèle cependant moins stérile qu'on pourrait croire lorsqu'on s'interroge sur le type de raison - il vaudrait mieux dire sans doute : de rationalité ou d'activité rationnelle - qui est à l'œuvre dans ce " penser ». En outre, en se précisant, elle marque davantage sa différence avec les sciences sociales du religieux. Peut-être cette différence en effet tient-elle autant au projet qui oriente leur recherche d'intelligibilité qu'aux catégories ou aux méthodes utilisées pour approcher le phénomène et le constituer en objet de science : la raison critique philosophique est une raison qui juge ou tend au jugement, qui cherche à connaître pour juger, qui estime le phénomène qu'elle cherche à comprendre, une raison qui cherche des raisons (et pas seulement des causes) - en quoi la philosophie de la religion exprime bien directement les intérêts de la raison. Or c'est sans doute ce que 
s'interdit par principe une science du religieux, en sorte que, à strictement parler, la philosophie de la religion n'est peut-être pas une science : elle continue d'être liée consubstantiellement à un projet évaluatif et pas seulement analytique.

Non pas qu'elle en serait toujours à penser - voire à vouloir élaborer - une religion dans les limites de la simple raison, mais encore une fois en ce qu'elle répond aux principes d'une raison déontologique. C'est particulièrement manifeste, on le verra ici, dans les développements, eux-mêmes divers, de l'épistémologie de la croyance dont le caractère normatif ne fait aucun doute: la tâche d'évaluer la rationalité des croyances religieuses qui guide le souci d'en comprendre la nature et le mécanisme exprime directement les exigences, fondatrices de la Modernité, d'un examen critique des croyances en général et de leur justification. On peut contester le modèle de rationalité qui porte ces exigences, contester les principes de «l'éthique de la croyance » qui guide cet examen ou la nature des devoirs épistémiques imposés aux croyants : cela prouve justement que la raison exprime dans la philosophie de la religion ses principes directeurs et qu'elle y vient simultanément les éprouver. Mais ce n'est pas moins le cas pour d'autres approches qui portent par exemple sur la signification des rituels, la contribution des représentations ou des expériences religieuses à l'élaboration de la pensée ou à son ressourcement, sa contribution, aussi, à l'autocritique de la raison, la légitimité et les moyens de sa participation éventuelle à l'élaboration des normes, ou bien encore le type de rapports qu'elle entretient avec les fondements du politique ou ses fins. Il n'est pas une seule des contributions ici rassemblées qui ne manifestent les enjeux, et même les enjeux brûlants, d'une inspection philosophique de la religion, la nécessité de cet exercice et son ancrage dans les intérêts de la raison.

C'est ce qui fait en outre qu'elle ne peut prétendre « penser philosophiquement la religion » ou " penser les religions » sans s'interroger simultanément, et peut-être d'abord, sur la présence du religieux aujourd'bui. D'une part parce que, à moins de s'enfermer dans la recherche stérile d'une essence transhistorique de la religion - ce que du reste elle n'a jamais fait - ou de se condamner à mécomprendre le phénomène religieux en utilisant des catégories qui ont été forgées pour des réalités qui ne sont plus les nôtres, elle a pour tâche de comprendre ce qu'est la religion dans sa nature et son environnement, dans ses rapports avec les sociétés, dans ses types de manifestations, dans ses lieux ; mais d'autre part parce que cette présence même soulève, au sein de la modernité, des problèmes, ne serait-ce qu'au titre d'une interprétation globale de la modernité - et que la philosophie de la religion trouve sa légitimité à les traiter. On voit mal comment, ayant elle-même forgé, avec Hegel, et développé l'interprétation ambivalente de la modernité comme sécularisation, elle oublierait une question qui a pratiquement toujours accompagné son analyse ou sa compréhension du phénomène religieux. C'est d'ailleurs en ce sens que, qu'elle le veuille ou non, elle doit bien rencontrer à un moment ou l'autre la démarche des sciences 
sociales. Lassé sans doute, et à juste titre, des gloses sempiternelles sur le « retour du religieux ", on aurait plutôt tendance à éviter d'en faire une raison décisive de ses développements. Il faut bien reconnaître cependant que la présence du religieux, et les formes de cette présence, dans l'Occident sécularisé est toujours, d'une manière ou d'une autre la source des interrogations philosophiques, par lesquelles la modernité s'interprète elle-même, que ce soit en termes de sécularisation, de post-sécularisation ou pour en rejeter encore comme obsolètes les catégories.

L'embarras qu'on éprouve cependant à ainsi qualifier la nature de la philosophie de la religion par les intérêts de la raison qu'elle exprime à l'égard du phénomène religieux montre sans doute les ambiguités de son projet fondateur (ne serait-ce d'ailleurs que celles qui se manifestent dans la question de savoir si, dans le syntagme "philosophie de la religion ", le génitif est seulement objectif et non pas aussi subjectif...) et la diversité des approches qui dès le début l'ont caractérisée : il y a déjà presque un monde entre l'explication anthropologique de la croyance religieuse chez Hume et la science de Dieu que veut être la Religionsphilosophie de l'idéalisme allemand. Aujourd'hui, il est de bon ton, là comme ailleurs dans le champ philosophique, de réputer stériles et impropres les divisions vulgaires entre tradition " analytique » et tradition "continentale » en insistant sur la variété qui caractérise chacune de ces « traditions » ou, plus rarement, sur leurs convergences ponctuelles ; il n'empêche que les différences sont si visibles parfois que non seulement elles se muent en hostilité franche (ou en mépris à peine dissimulé), mais que la " philosophie de la religion » devient une dénomination vide qui cache des réalités sinon incompatibles du moins parfaitement hétérogènes.

En lisant les contributions qui suivent, on comprendra assez facilement qu'il est parfois difficile de faire dialoguer ces traditions entre elles : non seulement elles n'ont pas les mêmes objets en vue ni les mêmes préoccupations, mais bien souvent l'exclusion ou l'ignorance des autres peut paraître constituer un prérequis. Mais le paradoxe est assez plaisant que ce soit notamment dans une revue de sciences sociales que cette rencontre (on n'ose parler de dialogue) se fasse. Ce n'est pas tant qu'il n'y ait rien de mieux que d'aller se disputer chez les autres ou encore de se trouver un ennemi commun pour feindre de se réconcilier: c'est surtout qu'en s'exprimant sur ce terrain, elles sont aussi contraintes, ces traditions, à définir la cohérence de leur projet, la raison de leurs différends et finalement la manière unifiée dont il faut entendre ce qu'est la philosophie de la religion aujourd'hui.

On n'aura pas de mal à voir apparaître ici ces différences; elles recouvrent sans doute des paradigmes identifiables et concurrents, c'est-à-dire finalement des conceptions divergentes de la raison ou de la rationalité, divergences dont l'une des dimensions principales se situe justement dans le type de rapport qu'elle 
entretient avec la religion. Mais l'intérêt est surtout d'apercevoir comment ces différences découpent en problèmes spécifiques le champ des phénomènes religieux.

Un premier massif est évidemment constitué par l'examen de la croyance religieuse, mais ce massif lui-même laisse apparaître une grande diversité de traitements, lesquels ne relèvent pas tous, on le verra, d'une épistémologie normative d'inspiration " analytique ". La philosophie y retrouve bien explicitement l'une de ses tâches fondatrices, qui consiste à poser la question de la rationalité des croyances religieuses, question, on l'a dit, qui ne peut manquer d'être immédiatement réflexive puisqu'il s'agit tout autant de poser la question de la légitimité des critères et des exigences qu'on prétend mettre en œuvre dans une telle évaluation. Et nul doute que par là elle n'exprime explicitement, outre cet enracinement, une demande ou peut-être une inquiétude sinon vitale du moins et plus que jamais actuelle ; car s'interroger sur la rationalité des croyances religieuses revient également à interroger ce que les religions elles-mêmes entendent faire de leur rapport aux « raisons ». Mais dans ces questions la philosophie ne peut pas non plus faire l'économie d'une interrogation sur la nature des croyances en question, et donc sur sa façon de les comprendre ou justement de ne pas les comprendre, sur la pertinence en définitive de la catégorie même de croyance qu'elle manipule.

Ce faisant, elle renvoie à un projet qui n'est plus seulement celui d'une épistémologie normative des croyances, ni même seulement d'une analyse de la croyance religieuse par ses causes plutôt que par ses raisons (que ce soit dans le cadre d'une critique anthropologique classique ou dans celui, plus contemporain, d'une épistémologie naturalisée), mais bien celui d'une interprétation des phénomènes religieux dans laquelle les «croyances » elles-mêmes ne sont peut-être qu'une dimension, et peut-être pas la plus importante : l'expérience ou la vie religieuses sont difficilement réductibles aux seules « croyances » fondamentales qu'on leur suppose dans l'examen du "théisme ", pas plus que ces dernières ne «fondent » les pratiques. Du reste, cette sensibilité n'est pas réservée à la seule phénoménologie «continentale " puisque le domaine la philosophie de la religion de langue anglaise possède sa tendance à privilégier l'approche de la religion comme "way of life » dans un héritage wittgensteinien presque toujours explicite. Interpréter ne signifie cependant pas seulement les comprendre comme formes de vie, mais aussi rapporter ces formes de vie à la fois aux situations historiques et existentielles et aux rapports herméneutiques des hommes à leur propre existence. On déplace en tous les cas l'inspection philosophique vers les structures de sens produites et portées par le religieux, lesquelles se manifestent à même les modalités de la vie religieuse, depuis ses couches les plus informelles jusqu'à ses pratiques rituelles. Un autre massif pourrait être donc être caractérisé par l'étude interprétative des " actes » religieux - et ces actes peuvent être aussi bien des actes de langage - à la fois dans leur sens et leur épaisseur historique. 
La philosophie y rencontre les sciences historiques autant que l'anthropologie ou la linguistique. Mais elle s'y rencontre aussi elle-même, dans son rapport à une ou des sources "autres » du sens dans lesquelles elle a peut-être désormais la tentation de trouver une ressource pour penser " autrement » (autrement que selon sa propre histoire métaphysique) après avoir si longtemps cherché à s'en déprendre : ce n'est pas moins ce que la raison (philosophique) doit au religieux - et ce non pas dans son verrouillage onto-théologique mais au contraire dans son ouverture possible - qui est alors son objet actuel.

Enfin un dernier domaine pourrait être désigné sous le terme, qui nécessite éclaircissement, de "théologico-politique », dont on ne peut non plus nier qu'il s'enracine dans une demande d'explicitation urgente mais qui confirme également la centralité de la philosophie de la religion dans l'autocompréhension de la modernité. Car c'est bien d'abord à l'interprétation de celle-ci en terme de sécularisation et de déliaison entre le religieux et le politique qu'elle se consacre alors - ou plutôt se consacre-t-elle justement à dépasser l'hypothèse d'une déliaison simple : le «théologico-politique " indique le domaine des formes d'intrications ou de croisements multiples du religieux et du politique dans la modernité tardive dont il faut bien rendre compte par des paradigmes concurrents. À dire vrai, il ne semble même plus nécessaire de s'interroger sur l'hypothèse d'une "permanence du théologico-politique » (Lefort dans son célèbre article y mettait un point d'interrogation), à la fois dans la théorie politique et dans la structuration des sociétés elles-mêmes, mais bien plutôt de déceler la forme de cette permanence, c'est-à-dire sa signification et surtout sa tendance. De ce fait, ces paradigmes n'engagent pas seulement les formes et même les conditions de possibilité de la philosophie politique moderne, mais bien l'interprétation de la modernité elle-même - et là aussi, la philosophie de la religion, gouvernée par ce que l'on pourrait appeler la "raison politique ", entre en dialogue nécessaire avec les sciences sociales du religieux.

Vincent DELECROIX

Groupe sociétés, religions, laïcités GSRL - UMR 8582 (EPHE-CNRS) vincent.delecroix@ephe.sorbonne.fr 
